\title{
Le Parlement congolais post-élection dans le processus de construction de l'espace public : mythe ou réalité ?
}

\author{
Célestin Tshimande Tukala*
}

\section{Résumé}

Depuis 2007, la RDC dispose d'un Parlement issu des élections libres, pluralistes, démocratiques, transparentes et crédibles. Il est à pied d'œuvre depuis ce temps et, a eu à légiférer et à débattre des nombreuses questions dont les plus importantes nous semblent être la question de la nationalité qui a été à la base de deux conflits armées que le pays a connus. Malgré l'organisation des élections, la mise en place du Parlement élu et l'existence d'une loi sur la nationalité, la problématique de la nationalité demeure toujours. La loi elle-même n'est pas respectée par les gouvernants, qui font montre de l'extranéité. Cela a suscité des remous et a contraint le parlement à lancer un moratoire qui n'a jamais été appliqué. Le parlement s'est saisi de ce problème qui a fait l'objet d'un débat mais le parlement n’a trouvé aucune solution à cette question du moratoire.

Il y a aussi la question sur les violents incidents ayant opposé la police Nationale congolaise (PNC) et le Bandu Dia Kongo (BDK) à Matadi et les territoires de Luozi, Seke Banza et Lukula, en février et mars 2008. Accusé de s'être arrogé les pouvoirs de l'Etat dans plusieurs villages où la présence de la PNC était très faible, le Gouvernement a lancé des opérations pour restaurer l'autorité de l'Etat dans l'ensemble du Bas Congo. Ces opérations ont vite pris la dimension de l'usage excessif de la force, des exécutions arbitraires, la destruction systématique des églises et des maisons du BDK, ainsi que le nombre important d'arrestations arbitraires.

D'autres question émergent notamment la question du crash de l'avion 'Antonov' mettant à nu l'inexistence d'une législation rigide dans le secteur de transports aussi crucial pour la sécurité de la population et celle de la conven-

* Centre d'études politiques (CEP), Faculté des sciences sociales, politiques et administratives, Université de Kinshasa, RDC.

Email : celetukala2008@yahoo.com 
tion de collaboration entre la RDC et le groupement des entreprises chinoises. Il se dégage des débats en rapport avec les questions traitées au parlement, un manque d'intérêt manifeste pour la mise en route d'une véritable démocratie politique, économique et sociale. Cet exposé veut explicitement de manière concrète et adéquate aux cas singuliers, analyser la qualité des débats au parlement pour en savoir si elle peut concourir à la construction de l'espace public.

\begin{abstract}
Since 2007, the DRC has had a Parliament established by free, pluralistic, democratic, transparent and credible elections. It has been hard at work since then and has legislated and debated numerous questions, of which the most important seem to be the issue of nationality, which was at the root of both armed conflicts experienced by the country. Despite the organization of elections, the establishment of an elected Parliament and the existence of a law on nationality, the nationality problem still subsists. The law itself is not respected by the authorities, which are externally controlled. This has caused a stir and forced the parliament to launch a moratorium that was never enforced. Parliament addressed the issue, which was debated, but parliament did not find a solution to the issue of the moratorium.

There was also the issue of the violent incidents opposing the national police of Congo (PNC) and the Bandu Dia Kongo (BDK) in Matadi and the Luozi territories, Seke Banza and Lukula, in February and March 2008. The BDK was accused of assuming state powers in several villages where PNC presence was very limited. The Government therefore launched operations to restore the authority of the state throughout the Lower Congo. These operations soon took on a dimension of excessive use of force, arbitrary executions, systematic destruction of BDK churches and houses, in addition to a large number of arbitrary arrests.

Other questions emerge, notably the crash of the 'Antonov' airplane, which revealed the lack of strict legislation in the transport sector, which is also vital for the security of the population, and that of the cooperation agreement between the DRC and a Chinese business organization. It is clear from the debates on the issues discussed in parliament that there is an obvious lack of interest for the establishment of real political, economic and social democracy. This presentation explicitly seeks to fully and concretely analyse through specific examples the quality of debates in parliament to determine whether it can contribute to the construction of public space.
\end{abstract}

\title{
Introduction
}

Par espace public, nous entendons avec Habermas (1978), le fondement du champ symbolique constitué par l'interface entre la sphère publique (État) et la sphère privée (les citoyens éclairés). Le concept d'espace public dans l'entendement du philosophe allemand met en relief deux principes, notamment le principe de publicité et celui d'opinion publique.

Habermas (1980:20) assimile purement et simplement « l'opinion publique » soit à la majorité parlementaire soit aux partis majoritaires ou ceux de l'opposition. Ainsi, il rend le Parlement comme cadre institutionnel qui est 
le point d'ancrage où se construit la volonté collective. En tant qu'émanation de la population et du corps électoral en particulier, le parlement ou la représentation nationale apparait sans nul doute comme l'institution par excellence appelée à contribuer à la construction de l'espace public tel que nous l'avons défini ci-haut. Il est en effet non seulement le lieu d'élaboration des lois mais aussi un organe de démocratisation de la vie publique et de refondation d'une citoyenneté politique et économique renouvelée.

Depuis 2007, la RDC dispose d'un parlement issu des élections libres, pluralistes, démocratiques, «transparentes ». Il est à pied d'œuvre depuis ce temps et a eu à légiférer et à débattre sur de nombreuses questions dont les plus importantes nous semblent être :

- La question de la nationalité. En effet, cette question est importante parce qu'elle a été à la base des deux conflits armés que le pays a connus. Malgré l'organisation des élections et la mise en place du parlement élu ainsi que l'existence d'une loi sur la nationalité jugée non discriminatoire aujourd'hui, la problématique de la nationalité demeure toujours pendante. La loi en la matière n'est pas respectée par les gouvernants dont la plupart détiennent une double nationalité. Cela a suscité des remous et a contraint le parlement a lancé un moratoire qui n’a jamais été appliqué. Le parlement s'est saisi de ce problème qui a fait l'objet d'un débat, néanmoins aucune solution à la question de la double nationalité n’a été trouvée jusqu’à ce jour.

- La question sur les violents incidents ayant opposé la police nationale congolaise (PNC) et le mouvement Bundu Dia Kongo à Matadi et dans les territoires de Luozi, Seke Banza et Lukala en février et mars 2008. Ce mouvement a été accusé de s'être arrogé le pouvoir de l'Etat dans plusieurs villages où la présence de la PNC était très faible. Le gouvernement a lancé des opérations pour restaurer l'autorité de l'Etat dans l'ensemble du territoire du Bas-Congo. Ces opérations ont vite pris la dimension de l'usage excessif de la force, des exécutions arbitraires, de destruction systématique des églises et des maisons du BDK, ainsi qu'un nombre important d'arrestation arbitraire.

- La question du crash de l'avion «Antonov » qui a mis à nu l'inexistence d'une législation rigide dans le secteur des transports, un secteur pourtant crucial pour la sécurité de la population.

- Enfin, la question de la convention de collaboration entre la RDC et le groupement des Entreprises chinoises, censée donner de l'impulsion à l'exécution du projet électoral du chef de l'État, devenu désormais le programme du gouvernement : il s'agit des « cinq chantiers». 
Il se dégage des débats en rapport avec les questions ci-dessus traitées au parlement, un manque d'intérêt manifeste pour la mise en route d'une véritable démocratie politique, économique et sociale. L'absence d'enthousiasme dans le chef de la population et de confiance vis-à-vis du parlement, est la preuve que cette institution en qui elle avait pourtant placé sa confiance au début, est loin de remplir la mission qui est la sienne. Vu la qualité des débats, peuton considérer cette institution pourtant importante et nécessaire à la construction, à la consolidation de la démocratie de masse comme un véritable lieu de débat public et de publicité non acclamative, pouvant permettre au peuple de se faire une opinion publique et être capable de faire usage public de la raison?

Pour y arriver, nous avons subdivisée ce travail en deux points. Le premier montre comment est né le parlement et les différentes mutations ou transformations qu'elle a connues au fil de temps et le dernier essaie, à partir des données concrètes, de voir si le parlement actuel issu des élections de 2006 peut contribuer à la création d'un espace démocratique. Une brève conclusion vient clore cette réflexion.

\section{Le Parlement dans l'architecture juridico-politique}

Le sens moderne du mot Parlement vient d'Angleterre où dès le XIIIe siècle, il désigne les deux chambres qui affirment leur pouvoir face au roi Jean sans Terre. Le parlement est né donc originellement de la volonté des comtes et des barons, des représentants de l'Église et des villes, de consentir aux levées d'argent dont le roi estimait avoir besoin pour mener à bien sa politique. On conçoit dès lors le glissement naturel qui s'effectue vers un contrôle de l'usage de ces fonds, ce qui constitue l'amorce du pouvoir de légiférer et contrôler.

Guy Hermet et al. (2005:229) montrent que le Parlement n'est pas à l'origine une institution démocratique mais le représentant des seules classes dirigeantes; il va le devenir en Angleterre à l'issue d'un long processus évolutif qui consiste à élargir progressivement l'assise électorale de la Chambre des communes au XIXe siècle, tandis que parallèlement périt le pouvoir de la Chambre des Lords (Bill de 1911) demeurée de composition purement aristocratique malgré les nominations de nouveaux pairs. Aujourd'hui, sous des noms parfois différents, presque tous les pays ont un Parlement, généralement composé de deux chambres, dont l'une au moins (la Chambre basse ou Assemblée nationale) est élue au suffrage universel.

Un Parlement est investi d'une triple fonction : adopter le budget, élaborer la loi, contrôler l'action de l'exécutif. Selon les régimes politiques, ces compétences s'exercent selon des modalités différentes. Dans un régime parlementaire stricto sensu, le gouvernement, responsable devant la Chambre 
basse, parfois également devant la seconde chambre, est susceptible d'être renversé. En revanche il dispose alors d'importants moyens de peser sur le travail législatif : fixation de l'ordre du jour prioritaire, dépôt de projets de lois, questions de confiance et menace de démission ou de dissolution.

Dans les régimes à plus forte indépendance réciproque entre Parlement et pouvoir exécutif comme aux États-Unis d'Amérique, le Parlement a une plus grande capacité d'entraver les projets législatifs de l'exécutif et de lui résister, mais l'exercice de son contrôle ne peut déboucher sur la démission du président, sauf en cas de mise en œuvre de procédures pénales. Cependant la limitation des armes juridiques de l'exécutif sur le législatif est souvent suppléée par d'autres formes de pressions politiques, notamment individuelles sur les membres du Congrès.

Hermet et ses co-auteurs attirent néanmoins l'attention sur une certaine crise contemporaine de l'institution parlementaire. La complexification du travail législatif dans les sociétés modernes, à la fois en quantité et en technicité, ne permet pas toujours aux commissions parlementaires d'exercer leur travail d'examen dans les meilleures conditions d'expertise ; en outre, elles sont le plus souvent dépendantes des informations fournies par les services administratifs du ministre. Quant à la fonction de contrôle, l'existence des majorités disciplinées, s'ajoutant à la faiblesse ordinaire des moyens autonomes d'enquête, en limite beaucoup l'impact effectif.

À cet égard, précisent-ils, le développement, dans les régimes pluralistes, d'une presse d'investigation et d'une magistrature moins frileuse, a contribué à déplacer vers d'autres espaces que les parlements la fonction, essentielle en démocratie, du contrôle des détenteurs du pouvoir d'État. La réforme, concluent-ils, est donc en permanence à l'ordre du jour, dans de nombreux pays, autour de thèmes comme la rationalisation du travail législatif, la mise à disposition des parlementaires de moyens matériels accrus, d'élargissement de leur droit aux documents administratifs internes, enfin des pouvoirs d'enquête au profit de commissions parlementaires créées.

Ces précieuses remarques de Hermet concernent aussi la République Démocratique du Congo dont l'histoire politique montre clairement que face au pouvoir exécutif, le Parlement n'a pas eu à jouer son rôle de représentant du peuple à travers ses fonctions généralement classiques énumérées cihaut. Mais la situation ne se présente pas de la même manière dans les pays où la démocratie s'est déjà installée et où la démocratie parlementaire est une réalité vécue presque chaque jour et où enfin les Constitutions qui régissent la vie politique ne sont pas à la merci des chefs d'État et d'exécutifs qui peuvent les modifier à tout moment et se donner plus de pouvoirs au cours de leurs mandats. 
Par ailleurs, il existe aussi des faiblesses liées au Parlement lui-même et qui en font finalement un organe parfois déconnecté des élus et en qui ces derniers n'ont plus confiance. C'est justement le cas de l'Assemblée nationale et du Sénat actuels mis en place au lendemain des élections multipartites organisées en 2006 à la suite d'une longue période de transition politique déclenchée en 1990. En effet, l'analyse des données sociopolitiques et économiques de cette période postélectorale montre bien que ce Parlement n'est pas un organe susceptible de bousculer les vieilles habitudes et promouvoir un espace public. Il faut bien sûr remonter à la sociogenèse même de cette institution pour en comprendre l'évolution et les mutations institutionnelles qu'elle a connues au fil de temps et essayer de relever les points de rupture (s'il y en a bien sûr) et de continuité qui nous permettront d'asseoir nos conclusions sur des bases scientifiques solides.

\section{Le Parlement congolais : regard sur la période coloniale et postcoloniale}

Le Parlement congolais est un fait d'importation, il est le fruit de la loi fondamentale, c'est-à-dire de la toute première Constitution de la jeune RDC au sortir de l'indépendance, loi qui a été élaborée dans la métropole par l'autorité coloniale (la Belgique) et léguée à la colonie. Ce parlement était composé d'une Assemblée nationale et d'un Sénat, il était le fruit des élections transparentes, libres et démocratiques organisées par le pouvoir colonial et qui ont permis la mise en place du nouvel État et de ses institutions politiques qui avaient normalement vocation à promouvoir un espace public démocratique n'eut été les événements malheureux survenus à l'aube même de l'indépendance.

Ces premières élections traduisaient la volonté de la population tenue pendant longtemps à l'écart de la gestion de la chose publique de se doter d'une représentation nationale qui allait finalement légiférer sur des matières qui la concernent. Exclus en effet de la participation politique pendant la période coloniale, les congolais ont évolué dans un système autoritaire, à la fois raciste et humiliant. Pendant la période coloniale en effet, la RDC est régie à partir de la métropole. En tant que colonie, la RDC a été gérée par la Charte coloniale qui en fixait la structure politique et administrative.

Le statut politique du Congo belge ne provient pas directement de la Constitution belge comme nous le rapporte Roger Delvaux, mais elle est la résultante de l'article $1^{\text {er }}$ de cette Constitution, révisée en 1893. Les colonies, possessions d'outre-mer ou protectorats que la Belgique peut acquérir étaient régis par des lois particulières. C'est ainsi qu'est issue la Charte Coloniale ou Loi Coloniale, qui a été modifiée et complétée à plusieurs reprises. C’est cette Charte qui fixe les droits des Belges, des étrangers et des indigènes et 
qui détermine l'organisation et le fonctionnement de la colonie. À cet effet, la colonie était dirigée politiquement à partir de la métropole par le Roi luimême, les deux chambres législatives belges, le Ministère des Colonies et le Conseil colonial. La loi intervenait souverainement en toute matière, elle était l'ouvre des Chambres législatives, mais la Charte Coloniale avait donné au Roi le pouvoir législatif sur la Colonie, pouvoir qui était exercé par voie de décret. Le rôle du Roi au point de vue législatif était considérable et c'est avec la collaboration du Ministre des Colonies qu'il remplissait cette mission. C'est le Ministre des Colonies qui proposait des décrets en rapport avec la gestion de la colonie et qui les contresignait.

Le Conseil colonial collaborait aussi à la législation du Congo belge. Les projets des décrets élaborés par le Ministère des Colonies étaient soumis au Conseil colonial avec un exposé de motifs et cet organisme émettait ses avis sur ces projets. De plus le Conseil pouvait initier des actions allant à demander certaines mesures législatives et exprimer certains vœux qui étaient adressés au gouvernement. Le Conseil colonial faisait partie nettement du pouvoir législatif colonial.

Au niveau de la colonie, le Roi était représenté par le Gouverneur Général qui normalement était exclu de l'exercice du pouvoir législatif, parce qu'il n’avait que des attributions exécutives. Néanmoins, en cas d'urgence et exceptionnellement, le Gouverneur général pouvait prendre des mesures d'ordre législatif au moyen des ordonnances-lois qui n'étaient valables que pour une période de six mois. Quant au pouvoir exécutif, il appartenait aussi au Roi des belges qui l'exerçait par voie d'arrêtés contresignés par le Ministre des Colonies, ou exceptionnellement par le Ministre des affaires étrangères (Delvaux 1945:30-31).

En fait, dans cette architecture politico-juridique coloniale, c'est le Ministère des Colonies qui jouait un rôle très important et la plupart des politiques publiques étaient élaborées par ce Ministère qui a fonctionné dans la Colonie jusque vers les années 1958, c'est-à-dire pratiquement jusqu'à la veille de la décolonisation. La Charte coloniale fixait aussi les droits et devoirs des indigènes, et la lecture de cette loi montre que les noirs étaient exclus de la politique qui est restée une chasse gardée des belges.

Comme on peut s'en rendre compte, les lois de la colonie étaient prises au niveau de la métropole, c'est en fait la représentation nationale ou le Parlement de la puissance coloniale qui élaborait les lois devant être appliquées dans la colonie et qui étaient proposées par le Ministère des Colonies et le Conseil coloniale. On sait que la plupart de ces lois étaient discriminatoires et racistes, et qu'elles avaient eu pour conséquence la marginalisation politique des « indigènes ». Il ne faut pas en effet oublier que la RDC était une colonie 
essentiellement d'exploitation et donc toutes les lois élaborées par la Belgique n'avait d'autre but que de favoriser l'exploitation des ressources naturelles de la colonie et mettre politiquement au pas les citoyens qui n'avaient pas le statut de citoyens.

L'espace public colonial était donc un espace autoritaire, dictatorial et totalitaire qui faisait des blancs des citoyens, c'est-à-dire des gens détenteurs des droits et qui faisait des noirs pratiquement des esclaves, des êtres sans droits et donc exclus de la citoyenneté politique et économique. Tenus loin de la gestion de la res publica, les colonisés n'avaient pas accès aux informations en rapport avec la gestion de la colonie elle-même qui était pourtant leur pays, une colonie dont l'administration coloniale est restée entre les mains des belges aussi bien à l'échelon national que local.

Cet apartheid ou racisme politique s'observe même dans la distribution spatiale ou l'occupation des espaces territoriaux aménagés de manière à séparer à l'époque les belges qui eux faisaient partie de la société civile, alors que les noirs en étaient exclus. Pendant la période coloniale, la presse était aussi au service de la colonisation, mieux encore de la mission civilisatrice. C’était un organe qui véhiculait l'idéologie coloniale belge et dont l'autorité coloniale se servait pour exalter les soi-disant bienfaits de la colonisation dans une colonie où a régné la terreur commise par les fonctionnaires du Roi Léopold II (18851908) et qui n’a pas cessé de continuer malgré la remise de l’État Indépendant du Congo à la Belgique en 1908.

Même les organisations dites féminines étaient également au rang d'instruments utilisés par le pouvoir colonial pour dominer et exploiter. Cette façon d'organiser et de gérer la colonie trouvait son fondement dans la philosophie coloniale belge : le paternalisme politique. Selon les Belges, les noirs étaient des enfants, des grands enfants pour qui il fallait tout faire. Il appartenait à l'homme blanc d'identifier leurs besoins, leurs désirs et de les satisfaire et la plupart de ces besoins étaient d'ordre social et économique, les besoins d'ordre politique étant étrangers aux préoccupations des colonisés. L'asymétrie dans l'assignation des rôles et statuts sociaux et des droits socioéconomiques et politiques aux différentes composantes de la société coloniale est la caractéristique majeure du régime colonial.

Bref, le pouvoir colonial, par définition un pouvoir de répression, d'oppression et de négation des droits et de la dignité des noirs ne pouvait pas contribuer à la construction d'un espace public, d'une sphère publique démocratique ou démocratiser les rapports sociaux entre blancs et noirs. L'absence d'une institution parlementaire siégeant dans la colonie et légiférant pour le bien être des colonisés est telle que lorsque furent organisées les premières élections législatives libres en RDC, les congolais y ont participé 
massivement, comme ce fut encore récemment en 2006 après plus de quatre décennies d'absence d'élections et d'un parlement réellement élu par la population depuis la prise du pouvoir par le Maréchal-Président.

Le Parlement naissant en 1960 était donc appelé à être une instance de débats et de d'examen des problèmes qui concernent enfin ceux que l'on a qualifiés pendant longtemps d' "indigènes » et qui étaient des « orphelins politiques, sociaux et économiques » pendant toute la période coloniale faute d'avoir des véritables représentants dans les deux chambres législatives belges et au Ministère des Colonies où s'élaboraient les grandes politiques publiques de la colonie. Le Parlement postcolonial était celui qui devait contribuer à la formation de l'opinion publique nationale, élaborer des lois non discriminatoires pouvant contribuer à la justice sociale, à la réduction de la fracture sociale et rendre publics les actes posés par l'exécutif.

Le régime politique mis en place en 1960 par la loi fondamentale lui donnait encore un relief tout à fait particulier. En fait, le régime instauré en 1960 peut être qualifié « de régime démocratique parlementaire ». Le Président de la République était censé isolé de la conduite de la politique et le Premier Ministre issu de la majorité parlementaire, était le chef d'un gouvernement responsable devant le parlement. Il y avait donc dualisme de l'exécutif et c'est la majorité parlementaire qui était appelée à déterminer la politique de la nation. Mais comme le dit si bien Kabuya Lumuna (1999:163), les expériences connues au Congo à cette époque montrent qu'il y avait eu un régime de cohabitation parce que les deux membres de l'exécutif n'ont jamais été de la même famille politique.

Selon la loi fondamentale (juin 1960- aout 1964), la signature du Chef de l'État était suivie d'un contreseing ministériel. Quand le Chef de l'État signait, il y avait contreseing du Premier Ministre ou d'un autre membre du gouvernement. Quand c'est le Premier Ministre qui signait, il y avait contreseing du ministre compétent. Il faut faire remarquer que d'après la loi fondamentale, le contreseing pouvait venir d'un ministre quelconque et pas nécessairement du «ministre compétent ». Sous le régime de la loi fondamentale, le contreseing indiquait que la responsabilité de la décision prise incombait à l'auteur de ce contreseing. C'était le moyen de contrôler les initiatives du Premier ministre ou celles du Président de la République.

Ces pouvoirs énormes accordés au Parlement faisaient de cet organe politique une institution de premier plan dans le processus d'engendrement de l'espace démocratique en RDC. C'est donc tout un programme politique de mise en place d'une démocratie parlementaire qui était recherché par la loi fondamentale. Le caractère fédéral imprimé à la structure politique de l'État et qui accordait aux provinces une autonomie provinciale, avait le 
mérite de faire également des entités infra-étatiques décentralisées des organes de promotion des espaces démocratiques locaux et des libertés publiques au niveau de l'échelon local où les dirigeants sont très proches des préoccupations des populations.

Il faut d'emblée signaler que ce parlement n'a pas eu la chance d'exercer ses missions librement pour des raisons que nous allons évoquer très loin, mais l'examen des activités parlementaires de cette période est de nature à nous faire voir que les élus du peuple de 1960, malgré quelques manquements, étaient mus par un désir : celui de représenter réellement les électeurs. C’est l'unique parlement qui s'est illustré par l'initiation des motions de censure contre les membres du gouvernement accusés de ne pas remplir leurs fonctions comme il se doit.

On le voit d'ailleurs à travers ces motions de censure prises à titre illustratif. Nous sommes en 1962, sous le régime du gouvernement Adoula. En effet, le 13 avril, M. Mutshungu du Cartel Unebafi-MNC-L. déposait une motion de censure contre Bisukiro, ministre du Commerce. Ce dernier était accusé :

- de mettre en place une politique consistant à éliminer tous les chefs traditionnels influents du pouvoir pour les remplacer par des nouveaux chefs qui seraient membres de son parti ;

- de s'être investi dans des activités jugées incompatibles avec ses fonctions de ministre selon la loi fondamentale ;

- d’avoir détourné des devises envoyées à la province du Kivu dont il était ressortissant, devises qu'il a affectées au fonctionnement de son entreprise ;

- de s'être approprié un hôtel, etc.

Les députés initiateurs de la motion exigeaient donc l'initiation d'une enquête au Kivu en ce qui concerne les activités commerciales du ministre Bisukiro : sur l'imprimerie, sur la banque, sur le Parc National, sur l'exportation des marchandises sans paiement des droits de douane, sur les devises, sur la gestion des Banyarwanda dans les affaires publiques de la Province, sur les finances détournées ainsi que sur les 14000 000F en provenance d'Allemagne.

Trois jours plus tard seulement, une autre motion de censure était déposée sur le Bureau de la Chambre contre M. Pinzi, ministre des Finances. Il était reproché à ce Ministre :

- d'avoir autorisé la vente et vendu à son profit personnel le stock d'ivoire qui se trouvait à Matadi ;

- d'avoir fabriqué ou fait fabriquer des fausses quittances ; 
- de n'avoir pas présenté, dans le délai imparti, le rapport financier réclamé par la Chambre des représentants ;

- de s'être fait ministre touriste et commerçant ;

- de n'avoir pas obtempéré à l'ordre du premier Ministre lui enjoignant de regagner la République ;

- d'avoir participé, en refusant d'obtempérer à l'ordre du Gouvernement de rentrer à Léopoldville, à la destruction de l'Autorité.

Il faut signaler que la motion de censure contre le ministre Bisukiro fut approuvée le 16 avril par 58 voix, contre 6 non et 7 absentions, sans que l'intéressé ait été entendu. Et le 20 avril, la motion de censure contre M. Pinzi était approuvée à son tout par 50 oui, contre 5 non et 14 abstentions, après que le ministre se fut longuement défendu. Ces deux motions furent sanctionnées respectivement par des Ordonnances n 56 du 28 avril 1962 et $\mathrm{n}^{\circ} 57$ du 28 avril 1962 portant démission de leurs fonctions des membres du Gouvernement. Pendant cette période les parlementaires ont effectivement exercé leur rôle d'organe de contrôle du gouvernement à travers l'initiation de plusieurs motions dont certaines furent rejetées, comme c'est le cas de la motion de censure initiée contre le ministre du Portefeuille, M. Badibanga, qui fut rejetée par 31 voix, contre 31 et 9 abstentions.

Ces actions et diverses activités parlementaires qu'on ne saurait toutes évoquer dans ce texte, montrent qu'un travail de construction de l'espace public était en train de se faire et que le jeune État était en train de s'installer sur la voie d'une gouvernance politique et économique démocratique. Ce Parlement était aussi résolument engagé dans la défense de la sauvegarde des libertés fondamentales et des intérêts de la population. En témoigne cette proposition de loi portant suspension de l'exercice du droit de grève pendant 2 ans, du 16 avril 1962. Cette proposition de loi avait pour but :

- de permettre aux pouvoirs publics de régler dans un climat de paix et de compréhension, les multiples problèmes qui se posent et d'éviter que d'autres difficultés ne surgissent entre-temps ;

- de protéger les travailleurs contre les manœuvres et les entreprises de certains groupes qui voudraient faire de ces travailleurs des instruments inconscients de leurs propres intérêts.

L'exécutif qui était l'initiateur de cette proposition de loi estimait que le vote de cette loi par l'Assemblée serait une contribution effective du Pouvoir Législatif à la consolidation de la paix publique, à la stabilité de nos institutions politiques et à l'amélioration rapide et sans heurts de la situation économique si gravement compromise. Cette proposition de loi proposée par M. 
Dericoyard fut retirée à la suite de l'intervention de trois députés, en l'occurrence MM. Mukwidi, Colin et Nyembo.

Ces députés avaient en fait tenu une argumentation qui montrait que cette mesure n'était pas démocratique parce qu'elle était de nature à étouffer l'exercice d'un droit très important, celui de faire la grève. Le malaise social, disaient-ils, était réel et le premier devoir du législateur soucieux de sauvegarder les intérêts de la masse (n'oublions pas que c'est au nom du peuple et pour le peuple que nous légiférons) est d'éviter ou plutôt de faire disparaitre ce malaise social. Nous devons marcher dans le sens de ce que la masse demande, rappela Mukwidi.

Nous ne voulons pas, renchérissait-il, que le Congo se transforme en dictature où les libertés démocratiques essentielles (association, presse) seront bafouées. Attendu qu'une paix durable ne peut être fondée que sur la base de la justice sociale, et que la liberté d'expression et d'association est une condition indispensable d'un progrès soutenu, nous invitons les honorables députés à rejeter, compte tenu de toutes les considérations qui précédent, la proposition de loi relative à la suspension du droit de grève. Et comme dit cihaut, les débats publics menés au parlement à la suite de ces arguments et qui épousaient largement l'idée de l'usage public de la raison de Habermas, avaient débouché sur le retrait de cette loi.

Mais cette expérience démocratique a fait long feu. Déjà en 1960 en effet, Le Président Kasa-Vubu révoquait Lumumba, le Premier Ministre, malgré sa majorité parlementaire. Le même Kasa-Vubu ignorait Tshombe et son groupe, majoritaire au Parlement en 1965. Le premier conflit entre le Parlement et le Chef de l'État éclata donc quelques jours seulement avant la session ordinaire des Chambres. Selon la Constitution de 1960 en effet, la première session ordinaire des Chambres devait s'ouvrir de plein droit le premier lundi du mois de septembre.

Cette réunion qui se tenait juste après la scène des révocations mutuelles du Chef de l'État et du Premier Ministre intervenues le 5 septembre, était donc d'une importance incalculable pour la vie politique de la jeune nation. Le 13 septembre, les deux Chambres réunies en assemblée commune annulèrent les deux révocations et conférèrent les pleins pouvoirs au Gouvernement Lumumba. À partir de ce moment-là, le conflit prenait une autre tournure et se compliquait : il opposait alors le chef de l'État à la fois a un Premier Ministre ayant la confiance du Parlement et au Parlement luimême. Le 14 septembre, le Président de la République, Kasa-Vubu ajourna les Chambres pour un mois. Le soir de ce même jour, le Colonel Mobutu opérait son premier coup d'État en neutralisant toutes les institutions politiques y compris les Chambres jusqu'au 31 décembre. 
Cependant, comme le montre Bomandeke (2007:15), la réalité des faits va démontrer, deux semaines seulement après, que ce pronunciamiento visait plutôt le leader de la majorité parlementaire et partant, l'institution législative. Car le 29 septembre, le président Kasa-Vubu, soi-disant neutralisé, promulgua un décret-loi constitutionnel relatif à l'exercice des pouvoirs à l'échelon national. Cet acte qui créait le Conseil des commissaires généraux, attribuait à ce dernier l'exercice du pouvoir législatif et maintenait l'ajournement du Parlement.

Le deuxième conflit entre le Parlement et le Chef de l'État est celui survenu en septembre 1963. Comme nous le rappelle encore Bomandeke, lors de l'ouverture solennelle de la session de mars 1963, le Président de la République avait demandé aux parlementaires d'accorder la priorité à l'étude du problème constitutionnel. Une commission mixte fut créée pour répondre aux souhaits exprimés par le chef de l'État. Mais compte tenu du fait que plusieurs séances publiques des Chambres se tenaient au même moment, les travaux de la Commission constitutionnelle s'avérèrent très lourds et lents. Le 26 août, le Président de la République surprenait encore la nation en convoquant les deux Chambres en Assemblée Constituante. Selon l'ordonnance de convocation, celle-ci devait se réunir dès le 30 août et siéger pendant 100 jours.

Selon la logique du Chef de l'État, la session était extraordinaire et de ce fait avait un calendrier bien précis. Son souhait était de voir les prochaines élections, imminentes, se dérouler conformément aux dispositions de la nouvelle Constitution. Les parlementaires qui n'entendaient pas voir leurs marges de manœuvre réduites par le chef de l'État et qui voulaient en même temps plancher sur la question du budget et celle de la censure du gouvernement, rejetèrent l'agenda du Président de la République. C’est ainsi que les deux chambres réunies envoyèrent, le 18 septembre, une proposition de procédure et de compromis au Chef de l'État, par laquelle elles lui notifiaient de leur intention de poursuivre les travaux ordinaires.

À la suite de cette notification, le Président Kasa-Vubu toujours décida de mettre fin aux travaux, désigna une Commission extra-parlementaire pour l'élaboration d'un projet de Constitution qui devait être directement soumis au référendum. Tous les parlementaires regagnèrent donc leurs circonscriptions respectives. Lieu privilégié de débat public, instance de contrôle du gouvernement et d'élaboration des lois, le Parlement était mis au pas et donc réduit au silence. C'est cette absence de lieu de débat public, d'expression de la liberté d'opinion et lieu de pluralisme idéologique, qui contraindra les responsables des partis politiques dont les activités étaient suspendues et les leaders politiques, de recourir aux armes pour se faire entendre. C’est dans cette mouvance qu'éclatent les rébellions des années 1963 qu'il faut considérer ici comme des moyens d'expression des revendications 
politiques, mieux de restauration de l'institution législative comme espace de débat public, comme organe de démocratisation de la vie politique.

On se rappellera que pour n'avoir pas tenu compte des leçons de l'histoire, la RDC a vécu la même situation très récemment. Quand Laurent Désiré Kabila est arrivé en 1997, il a suspendu les activités des partis politiques, mis en hibernation le Parlement et nommé par Décret- loi des parlementaires à la lumière de ses propres principes. On s'est retrouvé avec un Parlement nommé, constitué d'une Assemblée nationale totalement acquise au régime issu de ce que l'on a appelé la « révolution ». Le résultat c'est que beaucoup de gens se sont estimés exclus de la politique et se sont arrangés pour rejoindre des mouvements rebelles issus en réalité d'actes d'agression perpétrés par certains pays de la sous-région des Grands Lacs. Et comme en 1960 ou 1963, les conséquences ont été toujours désastreuses.

Comme on peut le remarquer, la quête du gouvernement de dominer le parlement ou de se ménager un espace parlementaire favorable à ses désirs et caprices en vogue aujourd'hui en RDC, ne date pas d'aujourd'hui, elle a une histoire qu'on peut situer dans un passé presque lointain. Ce qui est intéressant dans ce registre c'est que déjà en plein régime parlementaire, le pouvoir exécutif n’a ménagé aucun effort pour violer la Constitution et instaurer des mécanismes informels de domination de l'institution législative aussi bien en 1960, en 1963 qu'en 1964, lorsque le même Président KasaVubu a ignoré Tshombe avec son groupe, pourtant majoritaire à la représentation nationale.

Avec la prise du pouvoir par Mobutu en 1965 par un coup d’État, la démocratie parlementaire déjà en difficulté et vacillante ou titubante se trouvera dans une situation où elle aura de la peine à se reconstituer parce que l'institution parlementaire que Mobutu met en place jusqu'en 1990, sera totalement modelée ou structurée par les idéaux du Mouvement Populaire de la Révolution (MPR) qui prendra l'espace politique en otage, y compris la société civile.

\section{L'institution législative sous le régime monocratique}

C'est le 24 novembre 1965 que le Colonel Mobutu accède à la magistrature suprême, grâce bien sur à un coup d'État du Haut commandement militaire. Du 24 novembre 1965 au 24 juin 1967, c'est la Constitution de Luluabourg qui était officiellement le cadre juridique de la République. C'est donc en 1967 que sera promulguée la nouvelle Constitution issue de ce que Mobutu a aussi appelé à l'époque : révolution. Entre-temps, il avait réussi à prendre des mesures qui l'ont placé au centre de la vie politique et qui avaient fini par parachever son coup d'État. Il faut d'abord rappeler qu'en 1964, la RDC se dotait d'une autre Constitution dite de Luluabourg qui fut la première à être soumise au référendum populaire. 
Cette Constitution démocratiquement votée par la population (bien que les partisans de Lumumba se trouvaient en rébellion à cette époque), n’a pas été appliquée à cause du coup d'État évoqué. Cette constitution était en effet considérée comme l'œuvre des Congolais eux-mêmes par rapport à la loi fondamentale héritée de la Belgique et qui aura été à la base des nombreuses crises que la RDC a connues se matérialisant par les conflits entre les deux pouvoirs : législatif et exécutif. Cette Constitution avait instauré un régime présidentiel, que Mobutu s’est arrangé à transformer en régime présidentialiste avant que ne fut élaborée la Constitution de 1967.

Trois grandes décisions repérées par Kabuya Lumuna et consacrées par trois ordonnances-lois, montrent déjà que Mobutu était sur la voie de l'autoritarisme depuis la prise du pouvoir en 1965. Il s'agit :

- de l'ordonnance-loi du 30 novembre 1965 par laquelle il s’octroya l'initiative de loi, et donc le droit de légiférer ;

- de celle du 9 mars 1966 par laquelle il s’octroya pleinement le pouvoir législatif, en précisant que les ordonnances-lois qu’il prendrait désormais seraient communiquées au parlement seulement pour information ;

- de celle du 24 décembre 1966 par laquelle Mobutu réorganisa les provinces, en réduisant leur nombre de 21 à 8 et en changeant la mission des autorités provinciales, qui devinrent des fonctionnaires et celle des assemblées provinciales qui devinrent de simples organes consultatifs. Avec cette décision, Mobutu qui était déjà Chef de l'État, Premier Ministre, Ministre de la défense, Chef des armées et de la sûreté, mais aussi législateur réussissait d’instaurer un régime monocentré.

Le processus de dictatorialisation du régime s'est poursuivi par la mise en place de la Constitution de 1967. Celle-ci institua un régime de concentration de pouvoirs, mieux de confirmation de concentration de pouvoirs parce que comme dit ci-haut, Mobutu avait déjà réussi à concentrer tous les pouvoirs entre ses mains en 1966. À partir de 1967, tous les décideurs agissaient par délégation, au nom du Président de la République. En ce qui concerne la légitimité, le président Mobutu, Chef du Parti, Chef de l'État, de l'exécutif, du législatif et du pouvoir judiciaire était aussi le « garant de la nation ». En fait le Chef de l’État devenait carrément la source de la légitimité même du pouvoir.

Il faut néanmoins faire remarquer que le parti unique, le Mouvement Populaire de la Révolution (MPR) a joué un rôle très important dans le processus de monocentration du pouvoir et de concentration des pouvoirs entre les mains du Maréchal Mobutu. En effet, à partir des années 1970, le 
MPR devenait l'unique institution politique du pays, en d'autres termes toutes les institutions du pays lui étaient subordonnées : aussi bien le législatif que l'administration locale en passant par l'exécutif, le judiciaire, etc. Un peu plus tard, le MPR deviendra encore le Parti-État, il se confondait en clair avec les organes de l'État. Avec cette innovation politique, le MPR devenait carrément l'unique source du pouvoir, mais il ne se confondait pas seulement avec l'État, mais aussi avec toute la société civile qu'il réprimait et opprimait.

L'idée selon laquelle le MPR était la nation congolaise organisée politiquement s'inscrivait en fait dans cette logique. Ce qui est bizarre c'est que ce parti unique était qualifié de démocratique par ses chantres. En effet, des efforts ont été déployés pendant toute la deuxième république par les idéologues du parti pour faire comprendre aux citoyens qu'il était possible d'avoir la démocratie au sein d'un régime monolithique et qu'il était aussi possible d'atteindre la démocratie par l'existence d'un seul parti politique monopolisant tout l'espace politique (Forcad 1984).

Pendant cette période, l'institution législative a été tout simplement reléguée à l'arrière-plan, son rôle était rempli par les organes du parti, et en particulier par le Comité Central et le Bureau politique du MPR. Sous la deuxième République, les institutions politiques de la RDC étaient : le Congrès, le Comité central, le Bureau politique, le Conseil Législatif, le Conseil Exécutif et le Conseil Judiciaire. Les trois premières institutions étaient des organes du Parti, elles étaient politiquement et stratégiquement plus importantes que les trois dernières institutions de l'État. Les décisions exécutées par le Gouvernement étaient prises, sous forme de résolutions, soit par le Congrès, soit par le Comité central ou le Bureau politique, le Parlement n'étant là que pour constater et entériner les décisions prises par ces instances. Même la révocation des hauts dirigeants de l'État était l'œuvre des organes du parti.

Ce que le MPR appelait les forces vives de la nation, c'est ce qu'on pourrait appeler aujourd'hui la société civile, mais une société civile très politisée et modelée par les idéaux du parti et regroupant des militants du MPR. À l'issue des congrès, il se dégageait des résolutions dans tous les domaines et le gouvernement était appelé à les exécuter (République du Zaïre/ MPR 1988). Entre-temps le Parlement se réunissait et on ne sait trop quelle était sa mission réelle tant elle ne se faisait pas sentir sur la scène politique. En fait c'est surtout lors des élections législatives que les gens se rappelaient encore que l'Assemblée était l'une des institutions politiques de la République. Mais les motions de censure y étaient quasi inexistantes, sauf à des rares exceptions.

Entre-temps, et c'est là le paradoxe des régimes autoritaires, le Parlement continuait à se réunir et à « légiférer » et même à adresser des questions orales aux membres du gouvernement. Un compte rendu de la réunion du 
Conseil Législatif (Parlement à l'époque) renseigne qu'en date du 4 juillet le Ministre des Finances, M. Kamitatu Masamba était reçu au Parlement pour réagir à la question orale qui lui a été adressée par les citoyens Commissaires du peuple (députés) Idambituo et Malu. La question orale portait sur l'endettement extérieur de la RDC et la deuxième portait sur le problème de l'exécution du budget de l'État.

Cette séance était présidée par le Président du Conseil Législatif de l'époque, Kalume Mwana Kahambwe. D’après le PV, sur 222 membres qui composaient le Conseil Législatif, 176 étaient présents, 8 s'étaient excusés, 37 n'étaient pas arrivés et un siège était à pourvoir. Le quorum étant atteint, le Conseil pouvait siéger valablement, conformément à l'article 37 du Règlement intérieur du Conseil. Après avoir informé les membres du Parlement sur le montant de la dette qui s'élevait à l'époque à 7.100.000.000 de dollars, le Ministre s'était longuement étendu aussi sur le problème de l'exécution du budget de l'État.

Un autre PV de la réunion de cette institution tenue le 2 juillet 1988 porte sur les questions orales adressées par le Parlement à trois membres du gouvernement, mais un de ces trois, en l'occurrence M. Nzege Alaziambina, s'était quand même présenté devant les « honorables » députés. Nzege était à l'époque Ministre de l'enseignement primaire et secondaire. Après avoir souhaité la bienvenue au Ministre, le président du Conseil législatif, le même Kalume avait justifié cette procédure par la Constitution qui lui donnait sa légitimité, ainsi que les directives et recommandations du Président-Fondateur du MPR, Président de la République, et les résolutions du $4^{\mathrm{e}}$ Congrès du MPR, les décisions d'État du Comité central.

On voit encore une fois qu'en réalité, le Parlement était tenu de suivre la ligne politique déterminée par le Parti et qu'il n'était pas du tout un organe autonome. La question orale adressée au Ministre Nzege venait du citoyen Kahemba, ce dernier interpellait le ministre sur :

- l'état délabré de l'infrastructure scolaire : absence d'installations ;

- l'insuffisance d'équipements scolaires et de matériels didactiques et de fournitures de bureau ;

- la corruption généralisée à tous les niveaux ;

- l'absence de conscience professionnelle ;

- la baisse du niveau de l'enseignement.

Dans tous les deux cas illustratifs ci-dessus, les Ministres ont été entendus sérieusement par les parlementaires et ont fourni leurs moyens de défense. Néanmoins, malgré ces questions orales, rares sont les ministres qui faisaient l'objet de motion de défiance. Tout s'arrêtait à ce niveau et après ces questions 
orales la situation financière tout comme la situation scolaire empiraient davantage. Au moment où Kalume était Président de cet organe, on a vu son effort de chercher à redonner à cette institution sa vitalité en évoquant sa mission de contrôle du gouvernement. Lors de la séance où le ministre de l'enseignement était reçu, le Président du Parlement avait évoqué les dispositions de l'article 90 de la Constitution, qui stipulait que « Le Conseil Législatif dispose du pouvoir de contrôle sur le Conseil Exécutif, le Conseil Judiciaire et les services publics de l'État ; Il exerce ce contrôle soit par la question orale ou écrite, soit par l'interpellation, soit par les Commissions d'enquête instituées en son sein », pour justifier sa démarche.

On voit là un effort que fournit une institution législative étouffée par l'exécutif et le parti pour essayer de faire son travail de représentation des électeurs. Il s'agit en effet d'un forcing politique dans un contexte peu propice à ce genre d'exercice auquel cette institution se livrait. $l$ faut en effet rappeler que le Parlement ne pouvait plus rester insensible à la crise sociale et économique qui avait atteint des dimensions inquiétantes. On est en 1988, et deux ans après c'était le déclenchement du processus de démocratisation, et donc l'heure était déjà au début des remous sociaux et l'État développeur autoritaire mis en route par Mobutu, avait perdu toute sa légitimité. Mais ces efforts seront toujours entravés par le gouvernement et le parti.

Ces deux instances étaient encore loin d'accorder au Parlement la liberté requise pour légiférer au nom du peuple. En réalité, à cette époque, le Parlement tente de se constituer en une institution pouvant promouvoir des débats publics, faire connaitre au peuple les actes posés par les membres du gouvernement pour lui permettre de se faire une opinion publique et être en mesure aussi de contrôler les "élus ", il recourt même aux articles de la Constitution ci-dessus pour justifier sa démarche, mais il ne peut y arriver parce que tout est mis en œuvre pour ne pas le lui permettre. Et on sait d'ailleurs que c'est dans cette mouvance que le Président Kalume a d'ailleurs trouvé la mort.

\section{La transition politique et l'institution législative au Congo}

En 1990, le Président Mobutu prononçait un discours historique par lequel il supprimait le rôle dirigeant du Parti, instaurait le multipartisme et le plurisyndicalisme. À la même occasion, il promettait la tenue d'élections pluralistes en 1993 et reconnaissait que la Constitution révolutionnaire de 1967, plusieurs fois révisée, devenait incapable d'encadrer les nouveaux phénomènes politiques. À partir de ce moment-là, toutes les institutions politiques du pays, dont le Parlement monolithique, étaient appelées à disparaître. Mais l'opposition, comme dans les autres pays d'Afrique, avait exigé la mise en 
place d'une Conférence nationale souveraine qui ferait la relecture de l'histoire du pays et mettrait en place un cadre d'où seraient issues les institutions de la transition.

Depuis 1990 jusqu'en 2006 en fait, le pays n'a pas eu de Parlement au sens classique du terme, c'est-à-dire des députés élus par la population et disposant d'un mandat électoral d'une durée bien déterminée. Tous les Parlements qui se sont succédé pendant cette période et qui ont porté des noms différents, étaient constitués des députés ou sénateurs nommés par le pouvoir exécutif, ce n'était pas en fait des lieux de débats publics. C'était des instruments forgés juste pour remplir la « rubrique politique : Parlement » pour qu'elle ne soit pas vide. En effet dans tous les pays du monde, il est devenu inconcevable d'avoir un État sans parlement et il faut faire avec.

Par contre, bien que n'étant pas issue des scrutins, la Conférence nationale a été un véritable lieu de débat public. En effet, dans l'histoire politique de la RDC, ce grand forum que le pouvoir de Mobutu a sérieusement combattu, aura eu le mérite de contribuer à la formation de l'opinion publique, de promouvoir à travers ses différentes discussions, l'usage public de la raison. C'est grâce à la CNS que les populations ont vu la nécessité de participer au débat politique. En fait, cette institution a d'abord mis à nu la mauvaise gestion de l'État par le gouvernement, mais aussi par les Parlements qui se sont succédé dans le temps, qui étaient incapables de légiférer pour le peuple. Jamais le peuple n'a eu droit à un débat public aussi franc et aussi démocratique que celui instauré par les participants à ce grand forum, même si certains dossiers très sensibles comme ceux relatifs aux biens mal acquis n'ont pas été ouverts à cause de multiples pressions exercées sur eux.

C'est aussi pendant cette période qu'est né le phénomène des parlementaires débout, des groupes de gens acquis à l'opposition non armée incarnée par l'Union pour la Démocratie et le Progrès Social et dirigée par Étienne Tshisekedi, et qui se réunissaient chaque fois à des endroits bien déterminés (généralement dans des rues de la capitale, dans des ronds points) pour s'informer mutuellement sur l'actualité politique, discuter publiquement de la chose publique, bref pour faire usage public de la raison.

Cette appropriation des espaces publics territoriaux par un public informé ou cherchant à s'informer restera un des épisodes les plus mémorables du processus de démocratisation, en ce qui concerne surtout l'éducation politique de la population. Il faut d'ailleurs faire remarquer que la presse écrite et même la presse audio-visuelle ont joué un rôle très important dans ce processus de démocratisation. Ces organes avaient consacré des rubriques entières à l'analyse des rapports et/ou déclarations politiques faites à la Conférence Nationale et les parlementaires debout s'en servaient aussi pour faire leurs débats et orienter leurs comportements politiques. Il faut d'ailleurs 
faire remarquer en passant que généralement les forums qu'on organise en dehors des parlements politiques sont ceux où se font des véritables débats publics, où les participants ont la liberté de mettre en relief les problèmes publics réels, les difficultés auxquelles les populations sont confrontées et qui appellent des solutions urgentes.

De tous les parlements de la transition, celui qui s'est constitué en 2003 et qui a légiféré pendant la transition post-conflit (2003-2006) et dont les membres n'ont pas été élus aussi, est d'une particularité qu'il faut évoquer dans ce travail. D'abord c'est un Parlement issu d'un Accord politique, l'Accord Global et Inclusif (AGI), qui a sanctionné la fin de la guerre. Il a la particularité d'avoir rassemblé des députés et sénateurs issus du pouvoir de Kinshasa, de la société civile, de l'opposition et surtout ceux des exmouvements politico-militaires rebelles. C'est donc une structure créée juste pour mettre fin à la guerre et essayer de contenter les politiciens qui s'étaient lancés dans des aventures de guerre, ainsi que d'autres qui étaient à Kinshasa à la recherche d'emplois.

La mission principale de ce Parlement était de produire des textes juridiques utiles pour la tenue des élections de 2006. Ella a aussi élaboré plusieurs lois et créé des commissions spéciales de contrôle du gouvernement, le plus célèbre étant bien sûr la Commission qui a planché sur l'examen des conventions signées en temps de guerre par les autorités rebelles et celles de Kinshasa et qui ont donné lieu à une espèce de prédation généralisée des ressources naturelles du pays. Ce Parlement ne pouvait pas disposer de tous les pouvoirs réels nécessaires pour contrôler l'action gouvernementale et même pour légiférer et donc représenter le peuple.

C’était une structure politique incontrôlée d'abord parce que composée de gens qui estimaient y être arrivés par des armes et qui n'avaient donc pas de compte à rendre à ce même peuple au nom duquel paradoxalement ils prétendaient agir. Ensuite, les députés ne pouvaient pas sanctionner les membres du gouvernement parce qu'ils n'en avaient pas des moyens juridiques et politiques nécessaires. Enfin, ceux qui étaient au gouvernement ou animaient les services publics générateurs des ressources avaient reçu de leurs entités respectives mission informelle de " collecter » grâce à leur positon, des ressources financières dont leurs partis avaient besoin pour préparer les élections de 2006.

Comme on le voit, depuis 1965, il n'a existé en RDC aucun Parlement capable de promouvoir un espace public de démocratie, un parlement capable de légiférer de manière autonome, un parlement en mesure de s'ériger en un lieu de débat public, un lieu de publicité non acclamative. Le Parlement actuel issu des élections pluralistes était censé être ce type de Parlement d'après la population qui était convaincue que l'absence de démocratie était 
due soit à des Parlements autoritaires du régime de Mobutu, soit à ceux pris en otage par les deux grandes familles politiques de la transition politique mobutienne (1990-1997) qui ont structuré l'espace politique ou encore à des parlements issus des conflits armés comme celui de 1997 et le plus récent qui a fonctionné de 2003 à 2006.

\section{Le Parlement post-élection et l'espace public}

L'objectif de cette étude comme nous l'avons dit dans la partie introductive est de savoir si le parlement actuel issu enfin des scrutins dits libres et transparents, est un véritable lieu de débat public, une institution politique pouvant enfin œuvrer ou œuvrant dans le sens de contrôler l'action gouvernementale et sanctionner ceux de ses membres inefficaces. Il s'agit de savoir si ce parlement est un lieu de construction d'une véritable démocratie, un organe différent de ceux qui l'ont précédé et décrits ci-haut, et pouvant se constituer en une tribune capable de défendre les intérêts de la population par la mise au pas ou en ordre des gestionnaires de la chose publique, conformément aux pouvoirs constitutionnels qui sont les siens.

D’après Habermas (1978:186), la vérité des lois n'est garantie que dans la mesure où une sphère publique élevée, en tant que Parlement, à la dignité d'organe d'État permet à des discussions publiques de faire apparaitre les nécessités pratiques qui répondent à l’intérêt général. Il faut en effet noter que dans la définition de ce qu'il appelle espace public, Habermas accorde une importance majeure au Parlement qui est appelé à jouer un rôle de premier plan dans la construction de la démocratie.

Nous allons, à partir de l'analyse des actions et activités menées par le parlement, essayer de voir si cette institution remplit les missions qui sont les siennes et pour lesquelles elle a été élue par la population. Nous avons estimé utile de nous intéresser à des événements graves qui ont contraint le parlement soit à convoquer un membre du gouvernement pour qu'il s'explique en plénière(le cas des violents incidents ayant opposé la Police nationale au mouvement Bundu Dia Kongo, la question du crash de l'avion et celle de la convention signée entre la Chine et la RDC), soit encore ceux pour lesquels le Parlement a eu à légiférer (le cas de la nationalité). Il s'gira de savoir si toutes les actions entreprises par le Parlement ont abouti à des résultats qui garantissent les intérêts du peuple ou seulement protègent les membres du gouvernement ou ceux du parlement.

\section{Le crash de l'avion dans un quartier populaire de Kinshasa}

En 2007, en plein quartier populaire de la ville de Kinshasa, un avion ayant échoué à décoller, a tout simplement fini sa course dans une commune proche de l'aéroport même, la commune la plus populeuse de Kinshasa. 
Pour rappel, la ville de Kinshasa est l'une des grandes métropoles africaines, elle a une population estimée aujourd'hui à plus ou moins 10.000.000 d'habitants et donc tout événement de cette nature ne peut qu'avoir des conséquences très dramatiques en termes de pertes en vies humaines. L'avion qui venait de s'écraser dans ce quartier populaire est de marque russe, un Antonov. L'annonce même de la marque de l'avion était déjà pour la population un objet d'irritation parce qu'en fait, dans l'histoire des nombreux accidents d'avion que la RDC a connus, ce sont ces mêmes avions qui ont toujours été mis en cause.

L'ampleur de l'événement, les images des êtres humains brulés vifs passant à la télévision et le fait même que cet accident qui rappelait celui survenu vers les années 1993 toujours à Kinshasa et qui avait aussi décimé des centaines de vies humaines, ne pouvaient laisser les députés indifférents. C'est donc fort de tout cela que le parlement va adresser une motion de défiance contre maitre Nkulu, Ministre d'État auprès de la Présidence de la République. Normalement, c'est le Ministre en charge des Transports et communications qui aurait fait l'objet de cette motion, mais sa révocation très précipitée n'a pas donné l'occasion au parlement de l'écouter. En fait, déjà à ce niveau, toute la communauté nationale s'était rendue compte de l'imposture.

Le Ministre d'État près la Présidence de la République a été finalement obligé de se rendre au parlement où les députés de l'opposition ont mis en relief les griefs mis à sa charge. En effet, au cours des débats très vifs et animés qui ont eu lieu au parlement, un des députés avait laissé entendre que le Ministre des transports avait pris une décision interdisant le vol des avions Antonov, de les clouer au sol jusqu'à ce qu'une enquête dûment faite permette de savoir s'ils pouvaient être autorisés à voler ou pas. Malheureusement, le Ministre Près la Présidence de la République s'était interposé et avait autorisé aux propriétaires de ces avions de les faire voler. Le Ministre d’État était donc appelé à fournir aux députés et à la population les causes réelles pour lesquelles il avait autorisé à ces avions de voler alors qu'ils étaient réputés être à la base de beaucoup d'accidents qui ont endeuillé plusieurs familles.

Après les moyens de défense fournis par le Ministre et qui ont été suivis aussi bien par les députés que par la population étant donné que ces débats étaient diffusés par la télévision nationale, il s'est clairement avéré que le Ministre en question s'était rendu coupable des fautes graves qui nécessitaient sa démission et la population était convaincue que lors du vote, compte tenu de la gravité de l'affaire, les députés allaient voter une motion de défiance l'obligeant à quitter le gouvernement. Dans les milieux académiques ou au sein de la société civile, nombreux sont d'ailleurs ceux qui ont crû qu'avant 
même le vote de cette motion, le Ministre allait, par élégance et pour respect pour les familles endeuillées, démissionner de lui-même comme cela se fait dans les véritables démocraties.

Des allusions étaient même faites aux ministres d'autres pays qui, en ce genre de circonstances, anticipent leurs démissions. Bref, la population comptait beaucoup sur la décision de ce Parlement qu'elle a elle-même élu et en qui elle continuait encore à placer sa confiance tant elle est animée par une personne (du moins l'assemblée nationale) qui semble, par ses interventions, être acquise à la cause de la population.

Mais c'était sans compter avec le caractère encore non démocratique de ce parlement que la population a finalement découvert le jour même du vote de la motion. En effet, malgré tous les débats qui ont eu lieu autour de ce grave incident, malgré les arguments de l'opposition démontrant que le Ministre était coupable, le parlement n’a pas voté pour le départ de ce Ministre qui est encore en fonction. La population a donc été surprise par cette décision inattendue. On est donc ici en présence d'un cas où le parlement s'érige en un lieu de débat public, ce qui est positif pour lui d'abord dans un premier temps, parce que l'on espère qu'au finish il va sanctionner le Ministre coupable compte tenu de la gravité de l'événement et du comportement du Ministre. Malheureusement, ces débats ressemblent à des bruits inutiles, à des exercices politiques sans objectifs précis et même à la limite à des instruments de marchandage ou de simple intimidation des ministres pour créer des occasions de corruption.

Par ailleurs, ce crash, tout comme ceux survenus avant et après, ont mis à nu l'inexistence d'une législation rigide dans le secteur des transports, ce qui expose les populations à une insécurité que le parlement ne peut accepter de voir perdurer. Une fois encore, malgré ce crash et tant d'autres, le parlement n'a pas encore mis en route un Code de l'aéronautique civile en RDC et le désordre continue toujours à caractériser le secteur des transports aériens si bien que pour beaucoup de passagers aujourd'hui, voyager à bord des appareils congolais qui desservent les lignes intérieures, c'est prendre un risque que n'importe qui ne peut se permettre.

Il faut aussi d'autre part signaler que les compagnies aériennes congolaises sont frappées d'embargo dans l'espace Schengen et le parlement ne fait rien. Entretemps, nos compagnies enregistrent des manques à gagner et cette situation n'est pas sans répercussions sur le vécu non seulement des agents mais de la population toute entière qui s'appauvrit davantage. Normalement si les députés étaient sensibles aux intérêts de leurs électeurs, ils auraient agi autrement. Tout porte donc à croire que le parlement actuel n’exerce pas un contrôle sur le gouvernement. 


\section{La question de la double nationalité : le fameux moratoire}

La question de la nationalité a été à la base des conflits armés que la RDC connus et continue à connaitre, elle méritait de ce fait pour le parlement une attention soutenue. Mais l'incurie de cette institution par rapport à cette question d'une importance indémontrable, étonne plus d'un. D'après la nouvelle Constitution, la nationalité congolaise est une et exclusive. Personne ne peut donc être détenteur d'une double nationalité (Art. 10). Mais il existe dans toutes les institutions du pays (Parlement, gouvernement, cours et tribunaux, etc.) des personnalités qui ont une double nationalité et qui exercent des hautes fonctions, ce qui est en fait une violation de la Constitution d'abord et de la loi sur la nationalité ensuite.

C'est l'Honorable José Makila qui avait soulevé la motion incidentielle au cours d'une séance plénière de l'assemblée nationale du mercredi 31 janvier 2007 par laquelle il proposait la mise en place d'une commission d'enquête chargée de vérifier des cas de double nationalité au sein de toutes les institutions de la RDC et d'en tirer toutes les conséquences. Sa proposition consistait à :

- procéder à la vérification des cas de fraude à la loi sur la nationalité, d'abord au sein de l'assemblée elle-même ;

- ensuite au sein d'autres institutions : gouvernement, cours et tribunaux, etc. ;

- à la suite de ces vérifications, procéder à l'invalidation des parlementaires pris en indélicatesse, prononcer la déchéance de leurs fonctions et révoquer tous les cadres qui prestent dans les services publics et qui ont une double nationalité ;

- enfin, transmettre l'ensemble des éléments à la justice pour dispositions utiles et condamnation des coupables.

Après les débats qui ont eu lieu autour de dette épineuse question, le Président de l'assemblée avait proposé un moratoire, c'est-à-dire un délai de grâce ou une période pendant laquelle on ne pouvait opposer cette loi à quelqu'un qui détient une double nationalité tout en supposant que l'intéressé est en train de mener des démarches pour se conformer à la loi. Jusqu'à ce jour ce moratoire est en cours et aucun parlementaire, aucun ministre, aucun haut responsable n'a daigné mener des démarches pour se conformer à la loi sur la nationalité et la Constitution. En réalité, ce moratoire était une manière de taire cette affaire.

Le parlement ne revient plus sur cette question pour légiférer et aujourd'hui on trouve dans toutes les institutions politiques du pays des responsables et dirigeants ayant deux nationalités. On est donc dans une 
situation où le petit peuple est obligé d'avoir une et une nationalité alors que les dirigeants en ont deux. On est donc là en présence de deux Congo : un Congo d'en haut, jouissant des privilèges qui vont au-delà même de la loi et de la Constitution et un Congo d'en bas composé de tous ceux qui n’ont pas droit à la parole, qui ne disposent pas des moyens politiques pour se défendre. On voit donc bien encore ici que le parlement actuel n'est pas une instance de promotion de justice sociale, un lieu de promotion de la démocratie. Contrairement donc aux apparences qu'elle affiche, ce n'est pas un lieu des débats publics francs destinés à résoudre les problèmes de la population.

\section{Le Parlement et le cas Bundu Dia Kongo (BDK)}

Il s’agit ici d'un épisode politique qui montre à la fois la détermination du parlement à contrôler le gouvernement et à défendre les intérêts du peuple et son incapacité pourtant à apparaître comme une véritable institution de sanctions et devant tirer parti des leçons pour légiférer en faveur de la population.

En fait, Bundu Dia Kongo est un mouvement magico-politico-religieux dirigé par Ne Mwanda Nsemi, un député élu de la province du Bas-Congo où se trouve d'ailleurs implanté ce mouvement. Le fondement philosophique de ce mouvement créé en 1969 est de contribuer à la régénération et la restructuration de l'Afrique noire et des peuples noirs du monde entier, la remise en cause de l'espace géopolitique africain désorganisé par les occidentaux depuis la christianisation forcée, la traite négrière, la balkanisation par la conférence de Berlin, la colonisation et le néo-colonialisme (Potentiel 2005:10).

Au mois d'octobre 2007, ce mouvement s'est investi dans un activisme politico-religieux qui a débouché sur la déstabilisation de la province du BasCongo. En effet, depuis cette date, des tensions ont été enregistrées entre les membres du BDK et les autorités locales dans plusieurs villes et villages de cette province. La présence de la police nationale congolaise étant très faible dans les zones en conflit, le BDK s'est arrogé les pouvoirs de l’État dans plusieurs villages. Le rapport de la MONUC (2008:3) signale que des commandants locaux de la police auraient été tabassés par les membres du BDK à Kinkenge et à Mbanza Muembe, et après avoir menacé les autorités locales, le BDK aurait obtenu la libération de tous les détenus de la prison de Luozi. Les 24 et 25 février 2008, deux hommes auraient été brulés vifs par le BDK dans deux incidents différents à Kinkenge et à Bethelemi après avoir été accusés de sorcellerie par le BDK.

Le 28 février, le Gouvernement a lancé des opérations pour restaurer l'autorité de l’État dans l'ensemble du Bas Congo. Selon l'Inspecteur Général de la police nationale, les opérations avaient pour objectif initial l'arrestation 
des auteurs des meurtres de Bethelemi et Kinkenge, mais ont pris une envergure plus importante face à la « résistance organisée » du BDK. Selon le Ministre de l'Intérieur, les opérations visaient à restaurer l'autorité de l'État dans toute la province. Pourtant le nombre d'éléments et la composition de la force de la police déployée, l'étendue géographique considérable des opérations, le type d'armes et de munitions utilisées, l'usage excessif de la force, les exécutions arbitraires commises, la destruction systématique des églises et maisons du BDK, ainsi que le nombre important d'arrestations arbitraires peuvent montrer que les autorités auraient pu avoir pour objectif de réduire considérablement la capacité opérationnelle du BDK.

Ces événements ont contraint également le parlement à adresser une question orale au Ministre d’État chargé de la sécurité et décentralisation, mais aussi à Monsieur Ne Muanda Nsemi, chef de ce mouvement qui est de surcroit aussi député élu. Comme toujours, au cours des débats publics qui étaient aussi très animés et qui ont même fait la fierté de cette institution d'abord, le député a présenté ses moyens de défense, mais le Ministre qui a été accusé par l'opposition d'avoir outrepassé ses pouvoirs a été aussi entendu. Ne Muanda a accusé le Ministre d'avoir commis un génocide.

Le député Kiakwama de l'opposition, qui est intervenu à l'ouverture des débats publics sur la question orale adressée au Ministre, avait déclaré que l'opération à mener par la police devait d'abord et avant tout être une opération de police normale. Ceci impliquait donc : la collecte des renseignements, l'identification des auteurs présumés, l'arrestation des suspects, dans le respect de leurs droits, la présentation à la justice des prévenus et des éléments de preuve matériels réunis pendant une enquête à charge et à décharge, permettant, ou non de les condamner. Or à entendre les réponses du Ministre, dira Kiakwama, aucun de ces aspects essentiels du travail des policiers n'a été accompli. Evidemment, pour le Ministre, cette opération avait pour but de restaurer l'autorité de l'État comme l'indique son mot adressé au parlement sur les violences du Bas-Congo.

Les débats qui ont eu lieu en rapport avec cette question ont démontré clairement qu'au-delà des violences observées dans le Bas - Congo, se trouvait en fait un problème général qui concerne l'ensemble du pays. En fait, depuis la mise en place de nouvelles institutions issues des élections, le malaise social est réel, la crise économique s'est aggravée et les populations ont l'impression que finalement leur vote n'a servi à rien parce que ceux-là mêmes qu'elles ont élus pour défendre leurs intérêts vivent aujourd'hui dans l'opulence alors qu'elles croupissent toujours dans la misère.

Les revendications aussi bien d'ordre sociale qu'économique exprimées par le BDK à travers Ne Muanda Nsemi qui en est son chef lors de son intervention au Parlement, sont la preuve qu'il n'existe pas de démocratie en 
RDC aujourd'hui, que les véritables débats publics qui concernent la situation réelle de la population ne sont menés ni au parlement national ni dans les assemblées provinciales. C'est donc la légitimité de toute l'institution parlementaire qui est en quelque sorte remise en cause.

C’est ce que le député de l'opposition, Kiakwama (2008) a si bien exprimé lors de ses interventions : de quelle restauration de l'autorité de l'État parlet-on dans un pays où la carte d'électeur sert de carte d'identité, où les Ministres et autres dignitaires circulent dans des véhicules sans plaques minéralogiques, où les membres des comités de sécurité sont présentés avant les Ministres et Députés, où la solde du soldat est détournée par son propre officier, où les réunions des Institutions se tiennent à l'improviste ? De quelle restauration de l'autorité de l'État parle-t-on dans un pays où l'impunité est aussi certaine que l'arrivée des pluies et de la saison sèche ?, déclarait-il. C’est donc toute la question de l'absence de démocratie dont parlait le député ci-haut.

Curieusement, alors qu'il croyait mettre en cause seulement les membres du gouvernement, il oubliait qu'il remettait en cause également l'institution parlementaire dont il est lui-même membre et qui, depuis son investiture, a elle-même initié des actions allant dans le sens d'octroyer 6.500 dollars américains aux députés alors que les fonctionnaires qui gardent encore leurs dérisoires salaires sont souvent impayés ou payés avec un grand retard. Les actes comme ceux-là, posés par l'institution qui est pourtant censée représenter la population, servent d'exemples aux membres du gouvernement qui ne peuvent s’empêcher de se lancer dans la prédation.

\section{La convention de collaboration entre la RDC et le Groupement d'entreprises chinoises}

Il a été signé entre la RDC et la Chine, à travers la China Railway Group Limited Sinohydro Corporative, une convention minière et d'infrastructures stipulée comme suit :

Les parties reconnaissent et déclarent qu'en signant la présente Convention de collaboration, elles poursuivent la réalisation des objectifs suivants :

- Pour la RDC trouver les ressources financières nécessaires à la réalisation des projets d'infrastructures nationales estimées importantes ;

- Pour le Groupement d'Entreprises chinoises ; investir dans le domaine des métaux non-ferreux dans les territoires de la RDC ;

- Le groupement d'Entreprises chinoises s'engage à mobiliser et mettre en place le financement pour la construction des infrastructures en RDC (Le «projet d'infrastructures »); 
- Le financement sera remboursé par les revenus de l'exploitation minière tels que des gisements cupro-cobaltines situés dans la région de Kolwezi, actuellement inexploités, dont l’Entreprise publique la Générale des Carrières et des Mines, en abrégé « GECAMINES » est titulaire des Droits et titres miniers qui s'y rapportent.

Cette convention minière et d'infrastructures signée d'abord à la sauvette par le Ministre des travaux non en tant que Ministre agissant au nom de l'État, mais en tant qu'individu engageant tout un État, a fait l'objet des débats publics au parlement suite aux accusations des députés de l'opposition qui estimaient qu'elle a été mal négociée parce que les avantages que la partie chinoise tirait de cet accord n'étaient pas proportionnels aux services qu'elle entendait fournir à la RDC. Plusieurs interventions faites au Parlement ont montré clairement que cet accord était à long terme préjudiciable pour la RDC et que sans remettre en cause son importance, il était utile de le renégocier pour qu'il devienne un Contrat véritablement gagnant-gagnant.

Malgré tous ces arguments et en dépit des nombreuses mises en garde mises en relief par les députés en rapport avec la souveraineté de l'État à long terme, ce contrat a été adopté par le Parlement sans modifications. Tout le monde ne s'est pas empêché de constater que comme la plupart des conventions conclues entre l'État congolais et les partenaires extérieurs, cette convention est aussi un contrat léonin et peut être pire que cela, parce qu'il a été négocié par un individu qui l'a signé comme tel.

Comme on le voit, ces faits montrent que le parlement actuel est loin d'être une instance de construction d'une démocratie sociale. Certes, on peut y trouver quelques individus mus de bonne volonté, mais ils sont noyés dans une masse qui ne se fait aucun souci pour la population.

On peut expliquer cette tendance du Parlement à entériner les forfaits de tout acabit commis pas les membres du gouvernement par un facteur important. C'est le fait que le parlement actuel est dominé par une majorité en faveur du pouvoir en place. Cette majorité parlementaire est donc derrière le Président de la République et le Premier Ministre dont le parti et d'autres encore ont signé ce qu'on appelle aujourd'hui l'« Alliance de la Majorité Présidentielle », AMP en sigle. La Charte créant l'AMP stipule en son article 4 que les objectifs de l'AMP sont ente autres :

- Agir ensemble en vue de gagner l'élection présidentielle et les autres scrutins afin de gouverner sous le leadership du Président de la République dans une volonté commune de bâtir un État de droit ;

- Permettre l'irréversibilité et l'approfondissement des réformes structurelles et institutionnelles engagées sous la houlette du Président de la République en vue d'ériger, au cœur du continent africain, une 
société d'égalité de chances, marquée par une croissance économique forte, durable et socialement partagée et une économie moderne, dynamique et pourvoyeuse d'emplois, gages de succès dans la lutte contre la pauvreté.

En vue d'atteindre les objectifs sus-évoqués (qui sont au nombre de 8, nous n'en avons retenu que ces deux pour notre réflexion), les membres de l'Alliance s'attèlent à :

- garantir la libre expression des sensibilités politiques au sein de l'Alliance, gage de cohésion interne et d'efficacité ;

- gérer de manière concertée les grands défis liés à la bonne fin du processus de démocratisation et notamment ceux relatifs aux opérations électorales en cours, etc.

Cette majorité présidentielle ancrée dans l'AMP s'est donc donné comme objectif réel de défendre mordicus le gouvernement et de protéger ses membres jugés par tout le monde inefficaces aujourd'hui. Cette AMP est donc aujourd'hui à la base de la domination de l'institution législative par le gouvernement. Ses membres semblent être liés à l'AMP par une espèce de mandat impératif dont l'objectif est de sauvegarder l'alliance au détriment des intérêts de la population. Il n'est pas un secret pour personne que la population attendait la démission du gouvernement intervenue depuis peu, mais comme elle n'a pas des représentants valables au Parlement, le gouvernement a continué à commettre ses forfaits sans être inquiété.

\section{Conclusion}

Cette étude montre donc que depuis l'accession du pays à l'indépendance, le parlement n'a pas joué son rôle de représentant du peuple pour des raisons qui tiennent de manière générale à la tendance qu'a eu le gouvernement d'en faire sa caisse de résonnance. À part le tout premier parlement issu des élections de 1960 qui, par ses productions législatives et ses différentes motions de défiance caractérisées par la démission de certains ministres comme nous l'avons démontré dans les lignes développées ici, la plupart des parlements qui se sont succédés, sinon tous, n’ont pas été des instances de promotion d'un espace public au sens de Habermas et tel que défini dans la partie introductive de ce travail.

Certes, depuis la prise du pouvoir par Mobutu en 1965 et l'instauration du monopartisme, on a assisté à la mise en route des parlements monocolores et sans pluralisme d'idées. Cette situation s'est malheureusement poursuivie jusqu'en 1990, mais le pseudo-pluralisme politique instauré au lendemain du processus de démocratisation n'a pas ouvert le terrain à l'émergence d'un 
parlement capable de prendre en charge les missions constitutionnelles qui étaient les siennes. C'était d'ailleurs des parlements nommés qui n’avaient pas de compte à rendre au peuple. La Conférence nationale souveraine s'est présentée comme l'unique forum qui a offert un véritable débat public à la population, elle a contribué énormément la formation de l'opinion publique et à l'éducation politique de la population.

Aujourd'hui, il est fort regrettable que le Parlement tant attendu par la population, celui consécutif aux premières élections véritablement démocratiques libres et «transparentes » de la RDC post-1965, ne soit pas celui qui représente la population, qui élabore des lois justes, s'occupe des problèmes publics réels et surtout, qu'il ne soit pas celui qui contrôle l'action gouvernementale, qui rend publics les actes commis par les membres du gouvernement. Les données analysées dans cette étude montrent bien qu'on est encore loin d'avoir un parlement qui puisse contribuer à la promotion d'un espace démocratique au sens de Habermas.

\section{Références}

Delvaux, R., 1945, L'organisation administrative du Congo belge, Anvers : Éditions Zaïre.

Bomandeke, B., 2007, Histoire parlementaire congolaise, Kinshasa : Konrad Adenauer Stiftung.

Commission Électorale indépendante, 2006, La Constitution de la RDC, Kinshasa.

Convention de collaboration entre la République Démocratique du Congo et le Groupe d'entreprises chinoises : China Railway Group Limited Sinohydro Corporation relative au développement d'un projet minier et d'un projet d'infrastructures en RD Congo.

De Monstelle, A., 1965, La débâcle du Congo belge, Bruxelles.

Forcad/Institut Makanda Kabobi, 1984, Les grands textes du mobutisme, Kinshasa : Bibliothèque du Parti.

Habermas, J., 1978, L'espace public. Archéologie de la publicité comme dimension constitutive de la société civile bourgeoise, Paris : Payot.

Hermet, G. et al., 2005, Dictionnaire de la science politique et des institutions politiques, Paris : Armand Colin.

Kabuya, L. S., 1999, Notes polycopiés de cours de sociologie politique, Ire Licence Relations Internationales et Sciences Politiques et Administratives, Université de Kinshasa (inédit).

Kiakwama, K.K., 2008, Discours de clôture des débats sur la question orale avec débat adressée à Monsieur le Ministre d'État, Ministre de l'intérieur, sécurité et décentralisation, Kinshasa, 29 mars.

Kiakwama, K.K., 2008, Intervention durant l'ouverture des débats sur la question orale à Monsieur le Ministre de l’intérieur, Kinshasa, 23 mars. 
Ministre d’État, 2008, Réponses de son Excellence Monsieur le Ministre d’État, Ministre en charge de l'intérieur, décentralisation et sécurité à la question orale de l'honorable Kiakwama Kia Kiziki en rapport avec la secte Bundu dia Kongo, 26 mars.

Monstelle, A. de, 1965, La débâcle du Congo belge, Bruxelles : Leclerc.

MONUC/Division des Droits de l’Homme, 2008, Rapport Spécial. Enquête spéciale sur les événements de février et mars 2008 au Bas Congo, Kinshasa, mai.

Ne Muanda, N., 2008, Le génocide de 2008 au Kongo central, Kinshasa, 20 mars.

Potentiel, 2005, N³509, 16 aout.

République du Zaïre/MPR, 1988, Rapport au IVe Congrès ordinaire du MPR, Kinshasa, N'sele (présenté par le Secrétariat du Parti).

République du Zaïre/Conseil Législatif/MPR, 1988, Compte rendu analytique de la séance publique du Conseil Législatif du 2 juillet. Session ordinaire d'avril, Kinshasa.

République du Zaïre/Conseil Législatif/MPR, 1988, Compte rendu analytique de la séance publique du Conseil Législatif du 4 juillet 1988. Session ordinaire d'avril, Kinshasa. 
